\title{
Magnetotransport in C-doped AlGaAs heterostructures
}

\author{
B. Grbić* C. Ellenberger*, T. Ihn*, K. Ensslin*, D. Reuter ${ }^{+}$, and A. D. Wieck ${ }^{+}$ \\ * Solid State Physics Laboratory, ETH Zürich, 8093 Zürich, Switzerland, \\ ${ }^{+}$Angewandte Festkörperphysik, Ruhr-Universität Bochum, 44780 Bochum, Germany
}

\begin{abstract}
High-quality C-doped p-type AlGaAs heterostructures with mobilities exceeding $150000 \mathrm{~cm}^{2} / \mathrm{Vs}$ are investigated by low-temperature magnetotransport experiments. We find features of the fractional quantum Hall effect as well as a highly resolved Shubnikov-de Haas oscillations at low magnetic fields. This allows us to determine the densities, effective masses and mobilities of the holes populating the spin-split subbands arising from the lack of inversion symmetry in these structures.
\end{abstract}

Modulation doping in p-type GaAs/AlGaAs heterostructures was introduced with Be as a dopant on (100) structures [1]. Subsequently the integer 2] and the fractional 3| quantum Hall effects were observed in p-type material and transistor action in such devices was demonstrated [4]. Although the mobility could be improved to several $10^{4} \mathrm{~cm}^{2} / \mathrm{Vs}$ [5], the further developement of such devices remained limited, presumably because of the diffusion of Be dopants in the structure. The peculiar valence-band structure and the effects of inversion asymmetry were analyzed in detail [6]. A revival of research on p-type heterostructures started with Si-doping on (311) surfaces (for a review see Ref. 7). Ultra-high mobilities exceeding $10^{6} \mathrm{~cm}^{2} /$ Vs were achieved and such samples became relevant for the investigation of the metal-insulator transition in two dimensions $8,19,10,11,12$. In a series of pioneering publications by the Princeton group two-dimensional hole gases in GaAs were analyzed in detail by transport experiments. This included the investigation of tunable spin splitting [13], ballistic transport [13, 14], in-plane magnetoresistance in view of spin polarization of the system [15, 16], a detailed analysis of the low-field resistance in a perpendicular magnetic field [17, as well as an understanding of the Rashba effect [18] in these systems. An overview of the bandstructure of p-type GaAs is presented in [19].

Recently it has become possible to dope GaAs with $\mathrm{C}$ acting as an acceptor [20] on (100) substrates. Such samples have been patterned into Hall geometries and equipped with Ohmic contacts. Best results have been obtained for alloyed $\mathrm{In} / \mathrm{Zn} / \mathrm{Au}$ contacts. We have investigated a series of different samples at temperatures between $100 \mathrm{mK}$ and $4.2 \mathrm{~K}$. In the following we present results obtained at $100 \mathrm{mK}$ hole temperature on a sample with a mobility of $160000 \mathrm{~cm}^{2} / \mathrm{Vs}$ and a carrier density of $3 \times 10^{11} \mathrm{~cm}^{-2}$. The two-dimensional hole gas (2DHG) is located $100 \mathrm{~nm}$ below the surface. Figure 1 shows the magnetoresistance $\rho_{x x}$ and the Hall resistance $\rho_{x y}$. Pronounced integer quantum Hall plateaus and minima in the magnetoresistance at fractions of $4 / 3$ and $5 / 3$ document the high quality of the sample. The inset shows the regime at low magnetic fields. Two different oscillation periods are clearly visible. The two periods are related to two spin-split subbands, as has been previously shown for Be-doped (100) structures [2] and for Si-doped (311) samples [13]. This spin splitting can be attributed to the effective in-plane magnetic field $\mathbf{B}_{\text {eff }}$, which is induced by strong spin-orbit coupling due to structural inversion asymmetry [19]. It should be noted that $\mathbf{B}_{\text {eff }}$ varies in magnitude and orientation as a function of in-plane wave vector $\mathbf{k}$. Even though for a given $\mathbf{k}$ the states are spin polarized, after averaging over all occupied $\mathbf{k}$ states the total spin polarization in each of the two subbands vanishes. In the following we will denote the two subbands by spin up and spin down, having in mind the spin orientation of the states with respect to the direction of local effective magnetic field. Due to the different dispersion relations of the two subbands, the higher energy spin subband is more populated with holes than the lower energy spin subband for a given Fermi level. The difference of the two densities, $\triangle \mathrm{N}$, will be called carrier imbalance. For the data presented in Fig. [1 there are two times more holes in the higher energy spin subband than in the lower energy spin subband.

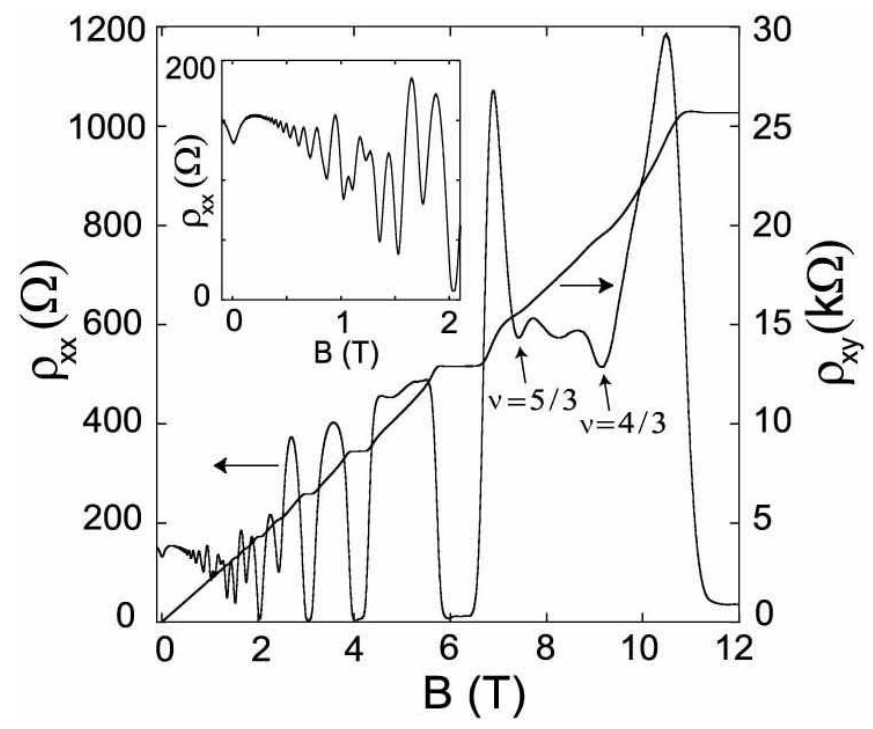

FIG. 1: Magnetoresistance of a p-type AlGaAs heterostructure. The minima related to fractional quantum Hall states are marked by vertical arrows. Inset: Blow-up of the low-field regime. A clear minimum of the magnetoresistance around $\mathrm{B}=0$ as well as a beating pattern in the Shubnikov-de Haas oscillations is observed.

With a homogeneous top gate $(\mathrm{Ti} / \mathrm{Au})$ the electron 


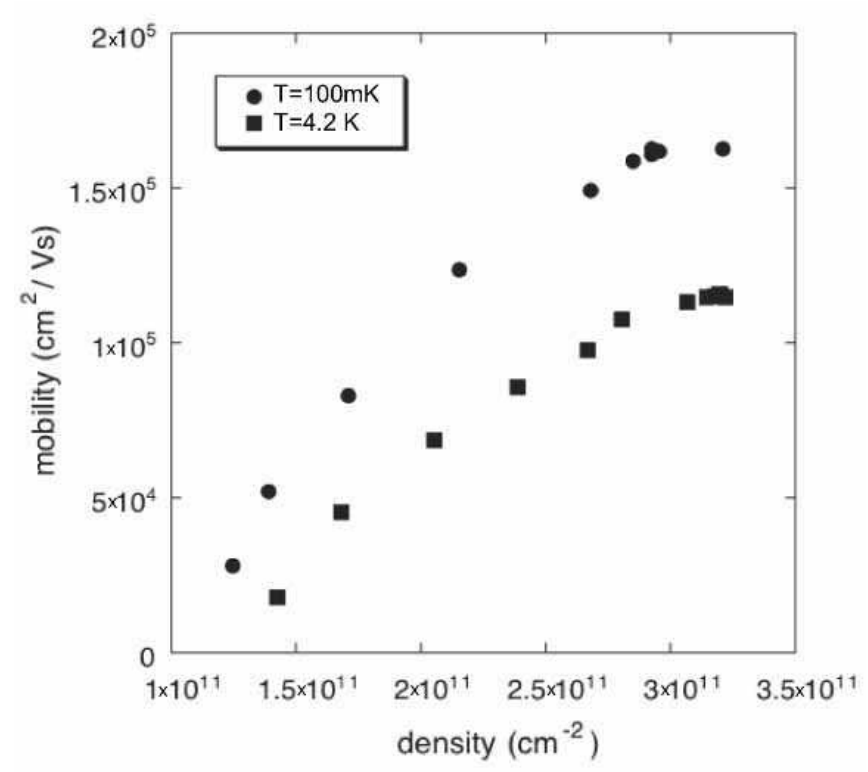

FIG. 2: Mobility versus carrier density tuned with a top gate voltage.

density and mobility could be varied, as shown in Fig. 2 for two different temperatures. The average mobility is determined from the resistivity at zero magnetic field, $\rho_{x x}$, not taking into account the two-subband occupancy. The mobility keeps increasing upon cooling of the sample even at temperatures below $1 \mathrm{~K}$ possibly because of reduced phonon scattering. It is apparent, that the mobility drops rather dramatically with decreasing carrier density and that pinch-off is determined by a mobility edge rather than by a vanishing carrier density.

After subtracting a second order polynomial background and multiplying with a smoothing window, the low-field $(0.3<B<2 \mathrm{~T})$ Shubnikov-de Haas oscillations have been Fourier-analyzed and the occupation of the two subbands was extracted. The corresponding data is plotted in Fig. 3 as a function of total density. The relative carrier imbalance increases with total density as expected from bandstructure calculations [19]. As the temperature is raised from $100 \mathrm{mK}$ to $1 \mathrm{~K}$ the carrier imbalance remains constant within measurement accuracy.

The mobilities of the individual subbands are analyzed under the assumption that the total conductivity of the system is composed of the conductivities of two individual subsystems. Intersubband scattering is not taken into account. The conductivity in the Drude model develops a parabolic B-dependence in the classical regime [21, 22], where Landau quantization is not yet important.

Figure 4 shows measured low-field magnetoresistance (solid line) together with the parabolic fit (dotted line). Taking the carrier densities of the two subbands as extracted from a Fourier analysis of the Shubnikov-de Haas oscillations as input parameters $\left(\mathrm{N}_{s}^{1}=1 \times 10^{11} \mathrm{~cm}^{-2}\right.$ and

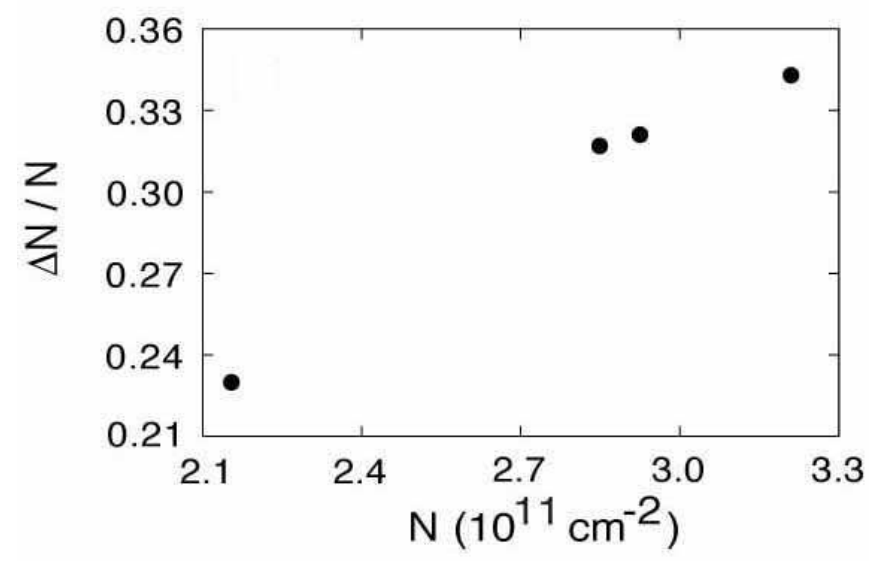

FIG. 3: Ratio of the carrier densities of the two subbands versus total density for a temperature of $\mathrm{T}=100 \mathrm{mK}$.

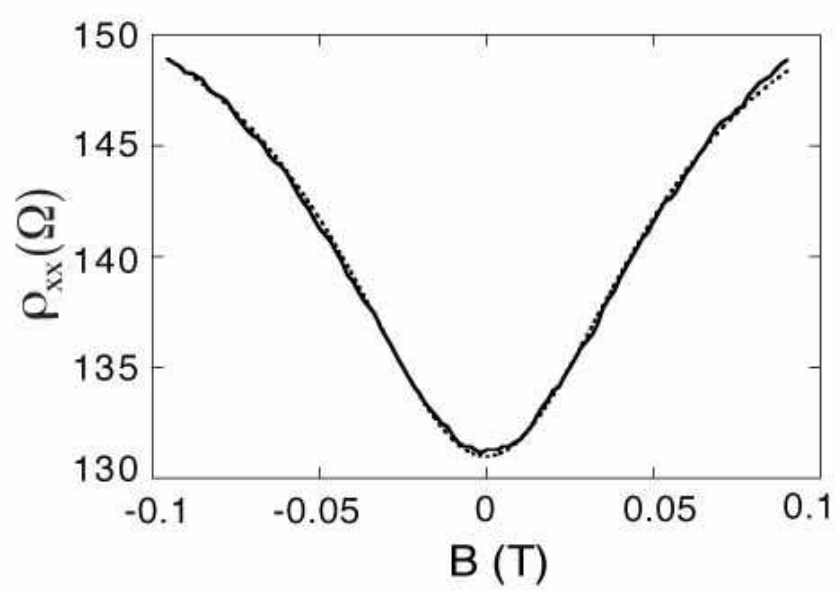

FIG. 4: Experimental low-field magnetoresistance (full line) and parabolic fit (dashed line) as described in the text.

$\mathrm{N}_{s}^{2}=2 \times 10^{11} \mathrm{~cm}^{-2}$ ) we obtain the mobilities of the two subbands as fitting parameters $\left(\mu_{1}=265000 \mathrm{~cm}^{2} / \mathrm{Vs}\right.$ and $\left.\mu_{2}=110000 \mathrm{~cm}^{2} / \mathrm{Vs}\right)$. The classical Hall effect becomes non-linear in the two-subband model. Using the above parameters we obtain satisfactory agreement between the measured and calculated curve.

As it is shown in Fig. 4 our 2DHG exhibits a very strong positive magnetoresistance at small perpendicular magnetic fields $(|B| \leq 0.1 \mathrm{~T})$. For the highest achievable densities in our system, $3.2 \times 10^{11} \mathrm{~cm}^{-2}$, the relative magnetoresistance, $\left(\rho_{x x}(0.1 T)-\rho_{x x}(0)\right) / \rho_{x x}(0)$ is $18 \%$. By reducing the density, at fixed temperature 100 $\mathrm{mK}$, the relative magnetoresistance continuously drops and reaches $4 \%$ at the lowest density $1.4 \times 10^{11} \mathrm{~cm}^{-2}$. Also, by increasing the temperature from $100 \mathrm{mK}$ to $1 \mathrm{~K}$ at fixed denisty $3 \times 10^{11} \mathrm{~cm}^{-2}$, the relative magnetoresistance drops from $17 \%$ to $5 \%$. This is in agreement with previous results [17], and represents clear evidence that 
there is a strong correlation between carrier imbalance in spin-split subbands with different mobilities and positive magnetoresistance.

From the temperature dependence of the amplitude of the low-field $\mathrm{SdH}$ oscillations the hole effective mass in the high-mobility spin subband is determined to be $m_{1}=(0.34 \pm 0.01) m_{e}$, which is in agreement with previous results for the effective mass in (100) plane [2]. Using the Boltzmann result for the elastic scattering rate 23. and the assumption that carriers from both subbands see the same scattering potential gives $m_{2} / m_{1}=\left(\mu_{1} / \mu_{2}\right)^{1 / 2}$. This relation, together with the previously obtained results for $m_{1}, \mu_{1}$ and $\mu_{2}$, gives $m_{2}=0.53 m_{e}$ for the effective mass of the low-mobility spin subband.

The quantum scattering time is obtained by fitting an envelope function to the low-field $\mathrm{SdH}$ oscillations, $\tau_{q}=$ $2.3 \times 10^{-12}$ s. The zero-field longitudinal resistance gives $\tau_{D}=3 \times 10^{-11} \mathrm{~s}$ for the Drude scattering time. The ratio $\tau_{D} / \tau_{q}=13$ indicates that a long range scattering potential is dominant.

By increasing the temperature from $100 \mathrm{mK}$ to $1 \mathrm{~K}$, the zero-field resistivity of the $2 \mathrm{DHG}$ increases by $6 \%$. This behavior, which has been interpreted as a metallic state of a 2 DHG 8, 19, 10, 11, 12], is another confirmation of the sample quality.

Our results demonstrate that C-doped (100) AlGaAsGaAs heterostructures show reasonable electronic properties comparable to the more established Si-doped (311) samples. Tuning of the carrier density with a homogenous top gate has been demonstrated. With a mean free path larger than $1 \mu \mathrm{m}$ such structures lend themselves for the fabrication of nanostructures either with split gates defined by electron beam lithography 24 or by local oxidation of the surface with an AFM [25, 26]. This would allow the realization of quantum dots and quantum point contacts with larger interaction strength, stronger spinorbit interaction and larger g-factor compared to n-type GaAs heterostructures.

We would like to thank Roland Winkler and Mansour Shayegan for valuable discussions. Financial support from the Swiss National Science Foundation is gratefully acknowledged.
[1] H. L. Stormer and W.-T. Tsang, Appl. Phys. Lett. 36, 685 (1980).

[2] H. L. Stormer et al., Phys. Rev. Lett. 51, 126 (1983).

[3] H. L. Stormer et al., Phys. Rev. Lett. 50, 1953 (1983).

[4] H. L. Stormer et al., Appl. Phys. Lett. 44, 1062 (1984).

[5] H. L. Stormer et al., Appl. Phys. Lett. 44, 139 (1984)

[6] J. P. Eisenstein et al., Phys. Rev. Lett. 53, 2579 (1984).

[7] A. G. Davies, J. E. F. Frost, D. A. Ritchie, D. C. Peacock, R. Newbury, E. H. Linfield, M. Pepper and G. A. C. Jones, J. Cryst. 111, 318 (1991).

[8] S. J. Papadakis and M. Shayegan, Phys. Rev. B 57, R15068 (1998).

[9] A. R. Hamilton, M. Y. Simmons, M. Pepper, E. H. Linfield, and D. A. Ritchie, Phys. Rev. Lett. 87, 126802 (2001).

[10] Y. Y. Proskuryakov, A. K. Savchenko, S. S. Safonov, M. Pepper, M. Y. Simmons, and D. A. Ritchie, Phys. Rev. Lett. 89, 076406 (2002).

[11] M. Rahimi, M. R. Sakr, S. V. Kravchenko, S. C. Dultz and H. W. Jiang, Phys. Rev. B 67, 081302(R) (2003).

[12] R. Leturcq, D. L'Hôte, R.Tourbot, C.J. Mellor and M. Henini, Phys. Rev. Lett. 90, 076402 (2003).

[13] J. P. Lu, J. B. Yau, S. P. Shukla, M. Shayegan, L. Wissinger, U. Rössler, and R. Winkler, Phys. Rev. Lett. 81, 1282 (1998).

[14] J. P. Lu, M. Shayegan, L. Wissinger and U. Rössler, Phys. Rev. B 60, 13776 (1999).

[15] H. Noh, Jongsoo Yoon, D. C. Tsui, and M. Shayegan, Phys. Rev. B 64, 081309(R) (2001).
[16] E. Tutuc, E. P. De Poortere, S. J. Papadakis, and M. Shayegan, Phys. Rev. Lett. 86, 2858 (2001)

[17] S. J. Papadakis, E. P. De Poortere, H. C. Manoharan, J. B. Yau, M. Shayegan, and S. A. Lyon, Phys. Rev. B 65, 245312 (2002).

[18] R. Winkler, H. Noh, E. Tutuc, and M. Shayegan, Phys. Rev. B 65, 155303 (2002).

[19] R. Winkler, Spin-Orbit Coupling Effects in TwoDimensional Electron and Hole Systems, Springer Tracts in Modern Physics, Volume 191, Springer-Verlag (2003)

[20] A. D. Wieck and D. Reuter, Inst. Phys. Conf. Ser. 166, $51(2000)$.

[21] H. van Houten, J. G. Williamson, M. E. I. Broekaart, C. T. Foxon, and J. J. Harris, Phys. Rev. B 37, 2756 (1988).

[22] G. Salis, P. Wirth, T. Heinzel, T. Ihn, K. Ensslin, K. Maranowski, and A. C. Gossard, Phys. Rev. B 59, R5304 (1999).

[23] J.H.Davies, The physics of low-dimensional semiconductors: an introduction, Cambridge University Press (1988).

[24] A. J. Daneshvar, C. J. B. Ford, A. R. Hamilton, M. Y. Simmons, M. Pepper, and D. A. Ritchie, Phys. Rev. B 55, R13409 (1997).

[25] R. Held, T. Vancura, T. Heinzel, K. Ensslin, M. Holland, and W. Wegscheider, Appl. Phys. Lett. 73, 262 (1998).

[26] L. P. Rokhinson, D. C. Tsui, L. N. Pfeiffer, and K. W. West, Superl. and Microstr. 32, 99 (2002). 